\title{
Celebrate contributions great and small
}

\author{
Obituaries paying tribute only to luminaries of microbiology risk overlooking the contributions of a diverse \\ community of researchers in our field. A new channel on the Microbiology Community provides a space for anyone \\ to write about the researchers that have influenced their work or career.
}

T he recent death of highly esteemed microbiologist Professor Stanley Falkow led us to revisit a conversation held several times previously among the editorial team at Nature Microbiology; whether to publish obituaries among our content. Taken at face value, this question may seem to have a straightforward answer. Falkow was a true leading light whose research and thinking have been fundamental in sculpting much of the modern microbiology field. Indeed, as very well described by Manuel Amieva in his recent piece for Nature (Nature 558, $190 ; 2018)$, Falkow's work in isolating and characterizing plasmids and the genes that they carried, on the nature of virulence factors and pathogenesis and the development of his 'molecular Koch's postulates', were foundational. Falkow also trained and inspired several generations of microbiologists who, together with Falkow, have established the field of molecular microbiology and greatly advanced our understanding of microbial pathogenesis over the course of the last half century. In short, if anyone deserves to make the cut for an obituary in our journal, surely Falkow would automatically qualify.

And yet, while the case for publishing a tribute to Falkow is clear, this particular conversation never simply rests at such a neat conclusion. For any publication, whether daily, weekly or monthly, assessing the career contributions of an individual and deciding whether to commission an obituary is a task of Escheresque complexity that is fraught with pitfalls and potential for political missteps. The main problem lies in defining criteria by which to decide to cover one individual and not another, and how these criteria can fairly be applied across a diverse field. How many ground-breaking discoveries does a researcher need to develop to reach the pass mark, an entire career's worth or can just one idea so daring and new that it leaves an indelible mark on the field be sufficient? Can a career spent making incremental but ultimately important steps count just as much? Does the microbiologist in question need to have trained and led others not just to follow in their footsteps, but to carve out new paths of their own, or is it sufficient to have focused solely on advancing their own research programme? Researchers rarely (if ever these days) work in isolation, and so one of the problems that afflicts the awarding of prizes such as the Nobel's also applies to obituaries; how to disentangle the contributions of collaborative efforts when deciding if an obituary is appropriate for one member of a team.

In addition to such scientific considerations, we also found ourselves asking whether by publishing obituaries we would, intentionally or not, find that we were paying tribute only to those individuals that had already received plaudits widely during their careers. In light of their efforts, such researchers may well have benefitted handsomely in terms of appointments at prestigious institutions, generous funding of their research over many decades and awards accrued. There will be many whose career achievements could justifiably have been judged as equal but for whom the limelight and wider acknowledgement of their efforts never materialized. Compounding this, obituaries will often (although by no means always) be considered 20 or 30 years after a researcher has retired, meaning that those who did not achieve a certain level of recognition during the active part of their career, or leave behind a field of trainees to carry a torch for their work, may be unfairly overlooked. Perhaps even more seriously, aside from those that pass away in their relative scientific youth, obituaries published now reflect the make-up of our field as it was 20-50 years ago. Consequently, while there are obituary-worthy microbiologists who also happen to be women and/or from under-represented minorities, the list of potential candidates is overall quite limited in its diversity and we risk paying tribute mainly to the often well-celebrated white men that dominated scientific faculties of the era.

Finally, there is the politically sensitive task of deciding who to approach to write an obituary. Certainly they need to have detailed knowledge of the individual in question, and this often means approaching friends, colleagues and collaborators (and sometimes even competitors) of the researcher in question. Yet, ensuring that an obituary is accurate and a fair description of the main contributions of their life and work can be challenging, especially outside of the strict scientific record where the use of anecdote and personal reflection is necessary to provide depth but can risk portraying the subject in the authors chosen light, which may be at odds with the experience of others.

As with the previous occurrences, our latest conversation on obituaries concluded with a decision not to include them as a content type published in Nature Microbiology, although we acknowledge that they can serve an important purpose in recognizing contributions to scientific fields (and understand how other journals considering these questions have come to different conclusions). However, out of these discussions grew an idea that we hope will address some of the issues described above. Using the platform provided by the Nature Research Microbiology Community, we have created a channel for what we are going to call 'Microbiographies' (https:// go.nature.com/2sWx818) that will provide a place for people to post recollections, anecdotes and thoughts on those people that have influenced their career. This need not be limited to posting only obituaries following a death, indeed we encourage the use of this channel to celebrate the contributions of any microbiologists that the author thinks suitable for praise, irrespective of whether they work at a prestigious institution or are lauded widely for their work. Is a microbiologist from your field or department retiring or moving? Perhaps post a microbiography to mark the occasion. We also hope to read stories of community member's personal heroes and those that have helped them along on their careers, no matter whether it was a giant shove or a little nudge. Importantly, the community is free to read and anyone can join and post to it, and so while we will from time to time be inviting microbiographies on particular individuals, the channel will be a democratizing space in which we hope to enable the field to celebrate microbiologists no matter the scale of attention that their contribution has previously garnered.

Published online: 26 June 2018 https://doi.org/10.1038/s41564-018-0193-8 